\title{
Non-geodesic orbital and epicyclic frequencies in vicinity of slowly rotating magnetized neutron stars
}

\author{
Pavel Bakala, Martin Urbanec, Eva Šrámková, \\ Zdeněk Stuchlík and Gabriel Török
}

Institute of Physics, Faculty of Philosophy and Science, Silesian University in Opava Bezručovo nám. 13, CZ-746 01 Opava

Czech Republic

email: pavel.bakala@fpf.slu.cz

\begin{abstract}
We study non-geodesic corrections to the quasicircular motion of charged test particles in the field of magnetized slowly rotating neutron stars. The gravitational field is approximated by the Lense-Thirring geometry, the magnetic field is of the standard dipole character. Using a fully-relativistic approach we determine influence of the electromagnetic interaction (both attractive and repulsive) on the quasicircular motion. We focus on the behaviour of the orbital and epicyclic frequencies of the motion. Components of the four-velocity of the orbiting charged test particles are obtained by numerical solution of equations of motion, the epicyclic frequencies are obtained by using the standard perturbative method. The role of the combined effect of the neutron star magnetic field and its rotation in the character of the orbital and epicyclic frequencies is discussed.
\end{abstract}

Keywords. Stars: neutron, X-rays: binaries, stars: magnetic fields

We assume the external gravitational field of slowly rotating neutron or strange stars approximated by the Lense-Thirring metric $(5,7)$, the magnetic dipole with the symmetry axis identical with rotation axis and infinitely conductive star interior implying force lines frozen into the star and dragged by its rotation (6). Solving the equation of motion for a charged test particles, we obtain orbital angular velocities $\omega \pm$ for corotating and counterrotating equatorial circular orbits (Fig. 1). Properties of the corotational and counterrotational circular orbital motion are naturally different due to the frame dragging of the external spacetime. The asymmetry is further amplified by the existence of two components of the Lorentz force with different behaviour. The Coulombic part of the interaction is induced by star rotation only, and is therefore independent of the test particles orbital velocity, while the orientation and amplitude of the magnetic part of the interaction induced by the orbital motion are strongly dependent on the orbital velocity.

Surprisingly, in the case of the counter-rotating orbits, starting from a critical specific charge $\tilde{q}_{\text {es }}$, the character of the electromagnetic interaction enables the existence of the electrostatic radius $r_{e s}(\tilde{\mathrm{q}})$, where properly charged particles are static relative to static observers at infinity. In such case, the gravitational attraction of the neutron star is compensated by the electric repulsion (Fig. 1).

The existence of epicyclic frequency corresponds to the condition of the stability relative to the appropriately oriented perturbations. Therefore, vanishing of the radial $\omega_{r}$ or vertical $\omega_{\theta}$ epicyclic frequencies determines the location of the marginally stable circular orbits (Fig. 2,3). We use analytic formulae for the radial and vertical epicyclic frequencies of a charged test particle in the presence of a general electromagnetic field derived and discussed by $(1,2,3,4)$. 

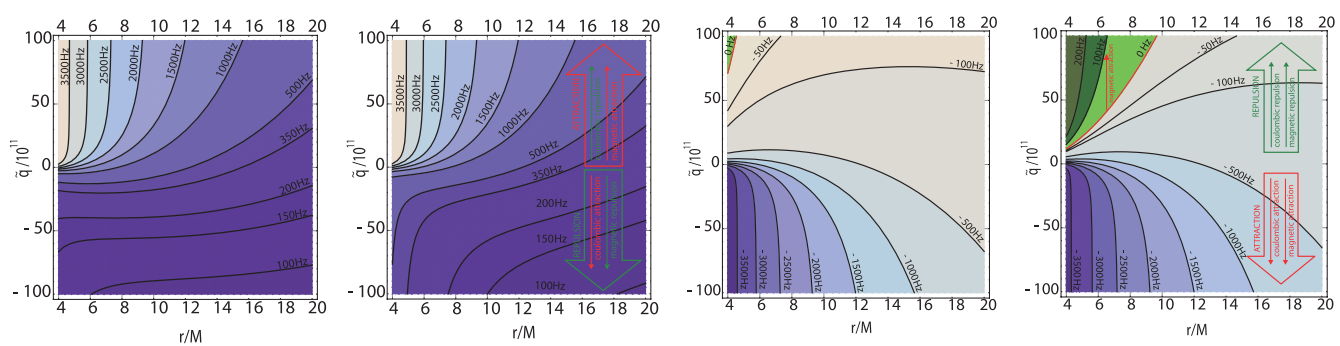

Figure 1. Contour plot of the orbital frequency $\nu=\omega / 2 \pi$ as a function of the specific charge $\tilde{\mathrm{q}}$ and the radial coordinate. Left: Corotating solution for spin $a=0.05$ and $a=0.3$. Right: Counter-rotating solution for spin $a=0.05$ and $a=0.3$.
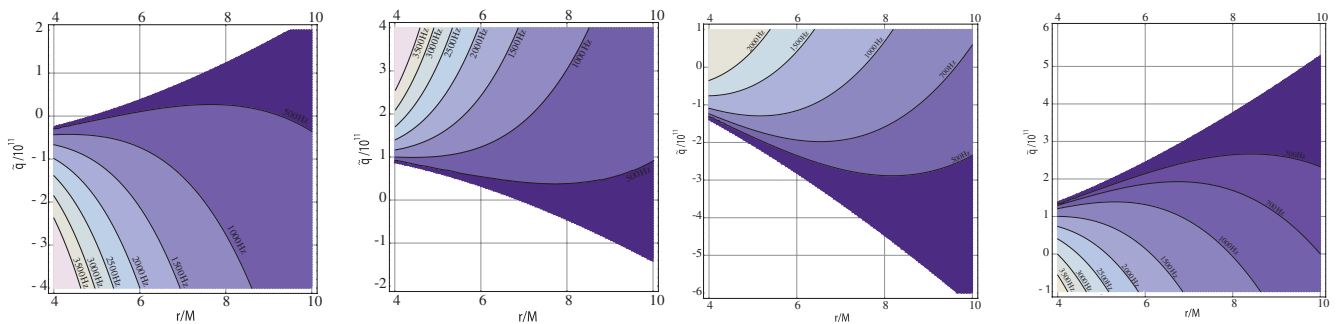

Figure 2. Left: Contour plot of the radial epicyclic frequency $\nu_{r \pm}=\omega_{r \pm} / 2 \pi$ as a function of the specific charge $\tilde{\mathrm{q}}$ and the radial coordinate for both solutions and spin $a=0.3$. Right: Same as the left panels, but for vertical epicyclic frequency $\nu_{\theta \pm}=\omega_{\theta \pm} / 2 \pi$.
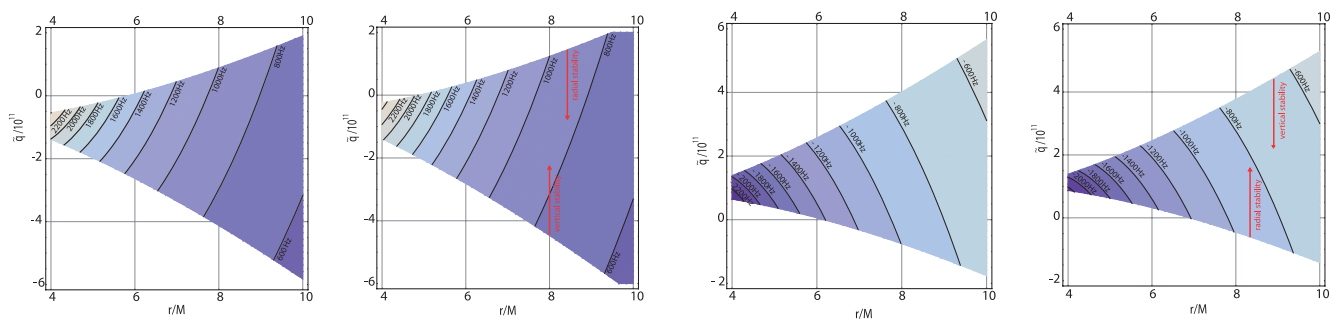

Figure 3. The region of stable circular orbits filled up by the contour plot of the orbital frequency $\nu=\Omega / 2 \pi$. Left: Corotating solution for spin $a=0.05$ and $a=0.3$. Right: Counter-rotating solution for $\operatorname{spin} a=0.05$ and $a=0.3$.

\section{Acknowledgments}

This work has been supported by the Czech grants GAČR 202/09/0772 and GAČR P209/12/P740. The authors further acknowledge the internal student grants of the Silesian University in Opava (SGS/1/2011, SGS/2/2011) and the project CZ.1.07/2.3.00/20.0071 "Synergy" in the frame of "Education for Competitiveness" Czech Operational Programme.

\section{References}

Aliev, A. N. \& Galtsov, D. V. 1981, GRG, 13, 899

Aliev, A. N. 2007, astro-ph/0612730v2

Bakala, P., Šrámková, E., Stuchlík, Z. \& Török, G. 2010, CQG, 27, 045001

Bakala, P., Urbanec, M., Šrámková, E., Stuchlík, Z. \& Török, G, 2012, CQG, 29, 065012

Hartle, J. B. 1967, ApJ, 150, 1005

Konno, K. \& Kojima, Y. 2000, Progress of Theoretical Physics, 104/6, 1117

Lense, J. \& Thirring, H. 1918, Phys. Zeitschr, 19 